Multiple disturbances classifier for electric signals using adaptive structuring neural networks

This article has been downloaded from IOPscience. Please scroll down to see the full text article.

2008 Meas. Sci. Technol. 19075106

(http://iopscience.iop.org/0957-0233/19/7/075106)

The Table of Contents and more related content is available

Download details:

The article was downloaded by: jajiang

IP Address: 140.112.94.195

The article was downloaded on 30/06/2008 at 15:01

Please note that terms and conditions apply. 


\title{
Multiple disturbances classifier for electric signals using adaptive structuring neural networks
}

\author{
Yen-Ling Lu ${ }^{1}$, Cheng-Long Chuang ${ }^{2,3}$, Chin-Shyurng Fahn ${ }^{1}$ \\ and Joe-Air Jiang ${ }^{2}$ \\ ${ }^{1}$ Department of Computer Science and Information Engineering, National Taiwan University of Science \\ and Technology, No 43, Sec 4, Keelung Rd, Taipei 106, Taiwan \\ ${ }^{2}$ Department of Bio-Industrial Mechatronics Engineering, National Taiwan University, No 1, Sec 4, \\ Roosevelt Rd, Taipei 106, Taiwan \\ ${ }^{3}$ Institute of Biomedical Engineering, National Taiwan University, No 1, Sec 4, Roosevelt Rd, \\ Taipei 106, Taiwan \\ E-mail: jajiang@ntu.edu.tw
}

Received 3 June 2007, in final form 6 April 2008

Published 17 June 2008

Online at stacks.iop.org/MST/19/075106

\begin{abstract}
This work proposes a novel classifier to recognize multiple disturbances for electric signals of power systems. The proposed classifier consists of a series of pipeline-based processing components, including amplitude estimator, transient disturbance detector, transient impulsive detector, wavelet transform and a brand-new neural network for recognizing multiple disturbances in a power quality (PQ) event. Most of the previously proposed methods usually treated a PQ event as a single disturbance at a time. In practice, however, a PQ event often consists of various types of disturbances at the same time. Therefore, the performances of those methods might be limited in real power systems. This work considers the PQ event as a combination of several disturbances, including steady-state and transient disturbances, which is more analogous to the real status of a power system. Six types of commonly encountered power quality disturbances are considered for training and testing the proposed classifier. The proposed classifier has been tested on electric signals that contain single disturbance or several disturbances at a time. Experimental results indicate that the proposed PQ disturbance classification algorithm can achieve a high accuracy of more than $97 \%$ in various complex testing cases.
\end{abstract}

Keywords: classifier, disturbance, electric signals, neural networks

(Some figures in this article are in colour only in the electronic version)

\section{Introduction}

At the present time, the quality of electricity supplies has become an important concern to electronic manufacturers and end-users. Poor electric power quality (PQ) might lead equipment and small electronic devices to malfunction or instability. The lifetime of electronic devices might also be shortened due to the resonance effect caused by harmonics or voltage flicker. Moreover, the disturbances caused by an enduser's devices will feedback to the power supplying systems, and further influence all other connected devices. Poor electric power quality can be attributed to power line disturbances such as wave shape faults. Thus, solutions to PQ problems must be found. Disturbance waveforms can be measured and recorded continuously using automatic monitoring equipment. Therefore, a large number of data can be collected, and manual inspection has become an unsuitable approach for detecting PQ events. Accordingly, a method that can automatically classify multiple types of disturbances in a PQ event must be developed. 
In recent decades, various approaches have been presented for automatically recognizing PQ events. Sentoso et al $[1,2]$ developed a PQ classification method based on wavelet analysis and a set of multiple artificial neural networks (ANN) to yield classification results with degrees of belief. Kaewarsa and Attakitmongcol [3] also utilized wavelet-transform-based neural network to recognize PQ events. Kaewarsa et al [4] used multiwavelet prefilters [5] to perform analysis on PQ disturbance waveforms. Chung et al [6] proposed a power disturbance classifier using a wavelet-based hidden Markov chain model. Sentoso et al [7] presented another approach based on the Fourier transform to analyze the disturbance waveform. Zhu et al [8] proposed a waveletbased fuzzy reasoning scheme to extract PQ disturbance features. Dash and Chilukuri [9] proposed an approach based on combining both the S-transform and Kalman filtering to detect short duration disturbances. He and Starzyk [10] utilized a self-organizing map (SOM) for automatically clustering power quality events into several classes. Shin et al [11] developed an algorithm to classify transient disturbances by using the instantaneous disturbance energy ratio, normalized instantaneous disturbance energy ratio, instantaneous frequency and instantaneous $K$-factor. Gerek and Ece [12] used a quadratic method with higher order cumulants to design power quality classifiers. Gerek and Ece [13] also performed power quality analysis based on an adaptive statistical method. Many other methods were proposed to detect power quality events, and to improve power quality using an adaptive filter [14-16]. Some software implementations were also available to analyze PQ disturbance waveforms [17]. These approaches can recognize the types of PQ disturbances with a specified accuracy. However, most of these approaches aimed at recognizing a single PQ disturbance in the measured electric waveform. Although some of these approaches can classify multiple disturbances, they provided operators with only an agreement level or confidence interval associated with the detected type of disturbances. The performance of such approaches might be limited in practical applications, because various types of disturbances are usually inherent in a PQ event in real power systems. For these reasons, this work develops a new classifier to recognize a PQ event with multiple disturbances and identify the critical levels of the detected disturbances.

In this paper, we address the implementation of the proposed PQ disturbances classifier in detail. The proposed classifier comprises an amplitude estimator, hierarchical wavelet transform scheme using Daubechies wavelets with 16 coefficients [18] and adaptive structuring neural network (ASNN). The primary reason for adopting ASNN in this study is its reconfigurable structure layout. Because the PQ events might contain different types of disturbances with diverse amplitudes, numerous examined waveforms will continuously be categorized into new training sample data packages. The reconfigurable feature of ASNN makes the proposed PQ events classifier with adaptability for the new examined PQ waveforms. Thus, the reliability of the proposed classifier can be enhanced for practical applications.

The rest of this paper is organized as follows. Section 2 introduces the types of PQ problems, presents the proposed scheme, and also offers an overview of the proposed method. Next, section 3 describes the proposed classifier that contains wavelet transforms, ASNN and its performance evaluation. Section 4 describes the model that we used to generate training and test data sets. Section 5 presents the experimental results of the proposed classifier. Conclusions are finally drawn in section 6 .

\section{System description}

\subsection{Types of disturbances in a $P Q$ event}

The power quality problems can be categorized into two main classes: steady and transient states. The following are the commonly encountered PQ problems considered herein to train and test the proposed classifier. These disturbances have been specified in detail in IEEE Std 1159-1995 [19].

Most of the conventional PQ event classification methods were tested by recognizing single types of disturbances in a measured electric waveform [1, 2, 7-9]. However, the field measurements indicate that various types of disturbances are typically contained in a PQ event simultaneously. Also, Chen and Sakis Meliopoulos [20] reported that the voltage and current waveforms in an electric power circuit are usually harmonic distorted and flicker modulated due to the intermittent operation of nonlinear devices with high power ratings. Therefore, this work proposes a new approach that can recognize various typical types of disturbances in a measured waveform and identify their critical levels.

\subsection{Overall configuration of the proposed classifier for multiple $P Q$ events}

This subsection presents the conceptual procedures that underlie the proposed classification algorithm for recognizing various types of PQ disturbances in a measured electric waveform. In this work, the classification of PQ events is conducted in three stages. In stage 1 , the amplitude estimator combined with the transient disturbance detector traces the rms value of the system voltage in a time domain to detect the swell, sag and interrupt disturbances. Then, in stage 2 the electric waveforms are transferred to a time-scale domain by using hierarchical wavelet transform to analyze the impulsive events. As in stage 3, the impulsive components of the wavelet coefficient of the waveforms are filtered out, and then the filtered waveforms are fed into ASNN for detecting harmonics and flicker. A conceptual block diagram of the proposed algorithm is depicted in figure 1. The inputs of the proposed classifier are electric waveforms that have been distorted by disturbances scattering around the power transmission lines, and the output is the types of disturbances contained in the electric waveforms.

In this work, the amplitude of the fundamental voltage can be estimated by the rms value of the observed waveform. Disturbances caused by voltage variations, such as the momentary sag disturbance, momentary swell disturbance and momentary interrupt disturbance, can be detected by tracing the increase or the decline of the rms voltage of the waveform in the time domain. According to the definitions given in 


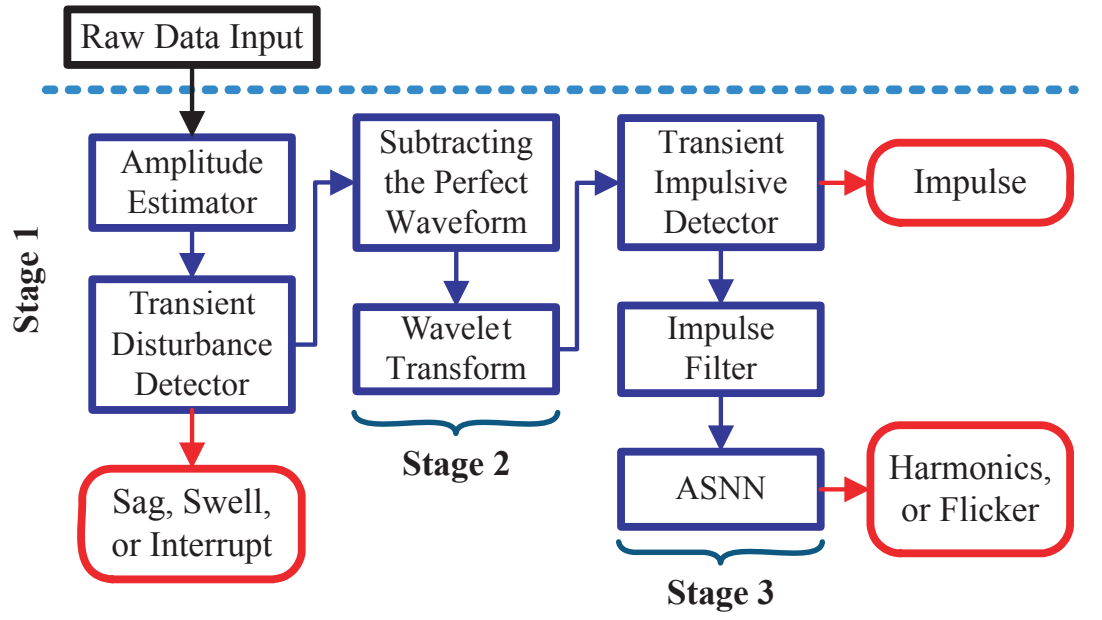

Figure 1. Block diagram of the proposed classification algorithm for multiple PQ disturbances.

the IEEE Std 1159-1995 [19], the steady-state disturbances can be detected by distinguishing if the value of the predicted amplitude falls in the specific interval of an abnormal range.

After the detection of transient disturbances is completed, the factor of voltage variations can be eliminated by subtracting an ideal sinusoidal wave with the estimated amplitude from the original distorted waveform. Therefore, the waveform of steady-state disturbances and impulsive transient disturbances can be extracted. The hierarchical wavelet transform is applied to decompose the waveform. An empirical rule is used to determine impulsive transient disturbances in the wavelet domain. Then, the factors of impulsive transient disturbances are removed using a mask filter. Finally, the standard deviations of the coefficients of the wavelet transform are fed to the input of the developed ASNN classifier, and the outputs are the types of steady-state disturbances classified by the proposed classifier.

\section{Components development of the proposed multiple disturbances classifier}

\subsection{Wavelet transform}

In this work, the wavelet transform is utilized to extract the most salient features of the PQ phenomenon. The wavelet transform effectively extracts the features of the PQ disturbances because it is very sensitive to irregular signals but insensitive to regular signals. Mathematically, the regular signal represents the background signal with the fundamental system frequency, and the irregular signals represent the noise or disturbances in the waveform. These irregular signals can be extracted and represented in terms of the wavelet coefficients for further processing.

Like other transforms, the wavelet transform comprises a pair of transforms from one domain to another [21]. Using wavelet transforms, the original signal is transformed from the time domain to the time-scale domain. This process decomposes the original signal into many signals with various resolutions. By recursively applying the wavelet transform to the lower frequency components of the PQ signal, a hierarchical wavelet transform can be obtained. For more detailed information regarding wavelet transforms, refer to $[22,23]$.

\subsection{Adaptive structuring neural network model}

Many popular classes of ANNs have been applied to pattern recognition and clustering analysis, such as multilayer perceptrons (MLP), Kohonen neural networks $(\mathrm{KNN})$, probabilistic neural networks (PNN), learning vector quantization (LVQ), counter-propagation networks (CPN), and adaptive resonance theory (ART) networks [24-27]. These ANNs perform well when they are with correct configurations, e.g. neuron size, network architecture, etc. However, there are no systematic criteria to provide guidelines for engineers to design an ANN architecture. Additionally, the architectures of traditional ANNs are fixed after their configurations have been specified. Thus, the performance of traditional ANNs might be limited once the status of the application is changed.

To cope with these problems, a learning algorithm that can automatically reconfigure the architecture of the ANNs is required. A novel ANN model, named 'adaptive structuring neural network (ASNN),' is developed to achieve this feature. The utilized ASNN can insert new neurons into the network to increase its learning capacity. On the other hand, it is also able to prune ineffective neurons and neural connections to save computational resources. Due to limited space, for the detailed technical information on ASNN, refer to [28].

\subsection{The proposed classifier}

Based on the conceptual block diagram depicted in figure 1, the detailed description for the data processing flow of the proposed classifier is provided in this section. The proposed PQ classifier consists of three major components: the amplitude estimator, hierarchical wavelet transform and ASNN. Figure 2 shows the detailed flow chart of the proposed classifier and its key functions are described as follows. 


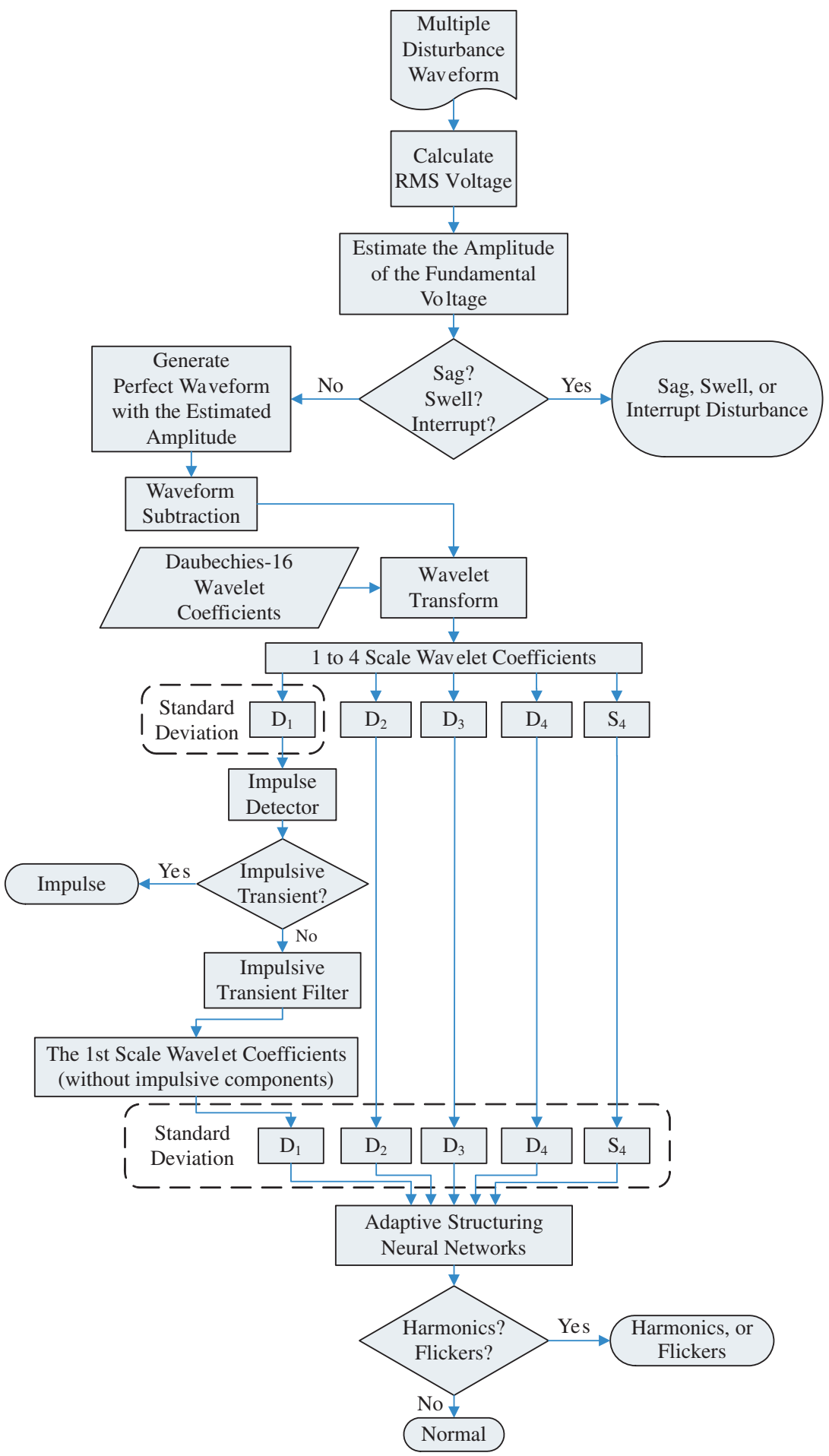

Figure 2. Flow chart of the proposed multiple PQ disturbance classifier.

3.3.1. Amplitude estimator. The wavelet transform technique has been widely exploited to extract the features from different types of electric signals. However, the wavelet transform is an unsuitable preprocessing method if the encountered disturbances are caused by a smooth increase or decrease in the voltage amplitude of an electric signal, such as the voltage sag or swell disturbance. Due to the insensitivity of the wavelet technique to irregular sinusoidal waves, these disturbances are analyzed and classified in the time domain rather than in the wavelet domain. Some waveletbased approaches $[1,2]$ tracked the local voltage minima and maxima of each cycle in the time domain to recognize a smooth increase or drop in the disturbance amplitude. However, the performance of the local voltage-tracking method is deteriorated when the electric signal comprises other kinds of disturbances. Hence, this work utilizes the rms (root-mean square) amplitude instead of local voltage minima and maxima to overcome the problem. 
In the following, we calculate the rms amplitude of a given waveform by

$$
\mathrm{rms}=\sqrt{\frac{\sum_{t=1}^{M}(f(t))^{2}}{M}},
$$

where $f(t)$ is the voltage sampled from the disturbance signal, and $M$ is the total number of sampling points in the duration of $1 \mathrm{~s}$. The reason for choosing $1 \mathrm{~s}$ as the duration time to calculate the rms voltage is that we assumed that the lowest frequency component contained in the signal is $1 \mathrm{~Hz}$, which can cover most of the possible frequencies that might exist in the electric signals of the power system. It is also adjustable to fit in with different requirements. For instance, if we want to consider the influence caused by a lower frequency component, say $1 / 2 \mathrm{~Hz}$, then we need $2 \mathrm{~s}$ of measured signal to calculate the rms voltage.

For some real-time applications, it is necessary to reduce the computational complexity of calculating the rms voltage, so the central processor would be able to process the other computational intensive algorithms such as the wavelet transform and neural network classifier. The computational complexity can be reduced by using the approximate rms voltage:

$$
\mathrm{rms}_{\mathrm{A}}=\frac{\sum_{t=1}^{M}|f(t)|}{M} .
$$

Once rms or $\mathrm{rms}_{\mathrm{A}}$ is determined, the estimated amplitude of the fundamental voltage can be transformed from an approximate rms voltage [19] by

$$
\mathrm{Amp}_{\text {Est_A }}=1.5725 \times \mathrm{rms}_{\mathrm{A}},
$$

where the estimated amplitude Amp Est_A represents the approximate rms value of the fundamental voltage waveform. For instance, we sampled a 1 p.u. (per-unit) ideal sinusoidal waveform with a sampling rate of $1800 \mathrm{~Hz}$, the value of $\mathrm{rms}_{A}$ is 0.6343. By applying equation (1), we can obtain an approximate rms Amp Est_A $_{\text {A }} 0.9974$ (p.u.). It is clearly seen that the estimation is simple, and the estimation result is also precise (the error is only about $0.26 \%$ ).

In accordance with IEEE Std 1159-1995 [19], the estimated amplitude Amp Est_A can be utilized to distinguish voltage sag, swell or interrupt in the time domain. Among these disturbances, only one of them can happen at a time. However, one or multiple other PQ events (e.g. impulsive transient disturbance, harmonic distortion and voltage flicker) may present in the disturbance waveform at the same time. Thus, after the amplitude of the fundamental frequency component has been estimated, the measured waveform is then analyzed by hierarchical wavelet transforms to identify other possible PQ events in the wavelet domain.

\subsubsection{Hierarchical wavelet transform. This study applies} the wavelet transformation to extract the disturbance features from the considered electric signals. Ideally, a sinusoidal waveform with the amplitude of Amp Est_A $_{\text {A }}$ is generated from power plant. But, there are many possible disturbance sources in the power system such that the measured electric signals usually do not exhibit ideal sinusoidal waveforms. Thus,

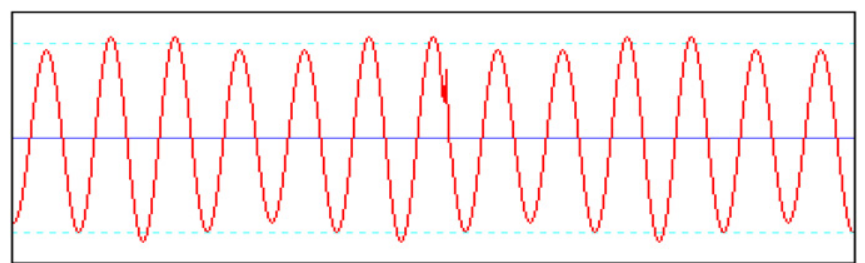

(a)

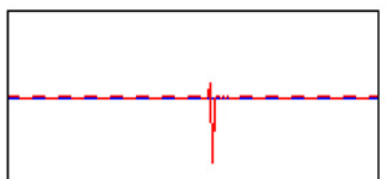

(b)

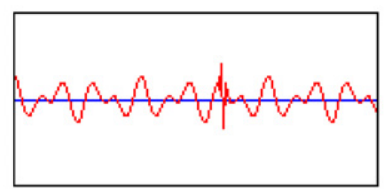

(c)
Figure 3. (a) An example of the voltage flicker and an impulsive transient disturbance; $(b)$ and $(c)$ the first scale wavelet coefficients of $(a)$ in the high-pass and low-pass bands, respectively.

disturbance signals can be obtained through subtracting an ideal sinusoidal waveform generated by a power plant from the original measured waveform. Following this step, the wavelet transform is applied to the disturbance signals to produce wavelet coefficients. The wavelet coefficients $D_{1}$, which contain the higher frequency components extracted from the measured electric signals, are utilized to determine whether disturbances are impulsive events or not.

The disturbance features are embedded in the decomposed high-pass and low-pass wavelet coefficients. The high-pass signal of the first scale decomposition is more sensitive to irregular variations than the other scales because it represents the signals with high-frequency components. This work utilizes Daubechies wavelets with 16 coefficients (DB16) to decompose the measured waveform. DB16 wavelets are utilized because they allow the hierarchical wavelet analysis to decompose the waveform into five components: $D_{1}, D_{2}$, $D_{3}, D_{4}$ and $S_{4}$ with desired frequency bands, which are 790$470 \mathrm{~Hz}, 480-235 \mathrm{~Hz}, 245-120 \mathrm{~Hz}, 125-60 \mathrm{~Hz}, 65-0 \mathrm{~Hz}$, respectively.

Accordingly, hierarchical wavelet transform is utilized to identify impulsive transient disturbances. As illustrated in figure 3(a), the disturbance waveform comprises the voltage flicker and an impulsive transient disturbance in the middle of the waveform. Figures $3(b)$ and $(c)$ show the plots for the high-pass and low-pass wavelet coefficients of the first scale decomposition, respectively. This example demonstrates that the decomposed signal in the high-pass band is very sensitive to the impulsive transient disturbance. From our investigations, it is found that the mean and standard deviation of the signal in the high-pass and low-pass bands of the fourth scale decomposition are a suitable choice. In this work, the mean and standard deviation of the signal in the high-pass band (i.e., $D_{1}$ shown in figure 2) are utilized to identify the impulse disturbance, which are respectively given by

$$
\begin{gathered}
\mu_{x}=\frac{\sum_{t=1}^{M / 2} D_{x}(t)}{M / 2} \\
\rho_{x}=\sqrt{\frac{\sum_{t=1}^{M / 2}\left(D_{x}(t)-\mu_{x}\right)^{2}}{M / 2}},
\end{gathered}
$$


where $\mu_{x}$ and $\rho_{x}$ are the mean and standard deviation of the wavelet coefficients in the high-pass band $\left(D_{1}\right)$ of the $x$ th scale decomposition, respectively.

For the detection of the impulsive transient disturbance, $D_{x}$ in equations (2) and (3) is the wavelet coefficient in the high-pass band of the first scale of decomposition, i.e., $D_{1}$. The impulsive transient disturbance event is identified based on the following empirical criterion.

$$
\forall t, \quad\left\{\begin{array}{lll}
H_{0}: D_{1}(t)=\mu_{1}, & \Rightarrow & \text { normal } \\
H_{1}: D_{1}(t) \neq \mu_{1}, & \Rightarrow & \text { impulse }
\end{array}\right.
$$

where the level of significance of the two-sided test is 0.2 ( 0.1 on each side). Therefore, if the condition $\left|D_{1}(t)\right| \geqslant$ $\mu_{1}+1.25 \rho_{1}$ is satisfied, an impulse transient disturbance event can be identified. The value of $1.25 \rho_{1}$ is chosen under the assumption that the possibility of making a decision to reject the null hypothesis $\left(H_{0}\right)$ when the null hypothesis is actually true is less than $20 \%$.

However, the impulsive transient disturbance may comprise various frequency components, which may cause the decomposed wavelet coefficients to become indistinguishable. Hence, the impulsive transient components must be removed from all scales of the decomposed wavelet coefficients to ensure that the performance of the ASNN classifier would not be influenced. The values of means and standard deviations of $D_{1}, D_{2}, D_{3}, D_{4}$ and $S_{4}$ are calculated using equations (2) and (3). Then, the values of means and standard deviations are input to ASNN to classify harmonic distortions and voltage flicker.

\subsubsection{Wavelet-based ASNN classifier. This investigation} proposes an ASNN-based classifier [28] to recognize the harmonic distortions and voltage flicker. The inputs of the ASNN are the standard deviations of the wavelet coefficients $D_{1}, D_{2}, D_{3}, D_{4}$ and $S_{4}$. These values are chosen as the input vectors because the features of the harmonic distortions and voltage flicker might distribute over all of the decomposition scales. An example is given in figure 4, which shows that the features of the disturbances are extracted in various scales of decomposition. The output of the ASNN is the classification result for harmonic distortions and voltage flicker.

3.3.4. Critical levels of $P Q$ disturbance events. After the types of disturbances are classified, the critical levels of the corresponding types of disturbances are also calculated for operators [29]. The critical level $C_{\mathrm{Amp}}$ for the voltage swell, sag and interrupt is defined below.

$$
C_{\text {Amp }}=\left|\mathrm{Amp}_{\text {Est }}-1.0\right| \times 100 \%
$$

In this work, the critical level of the impulsive transient disturbance $C_{\text {Imp }}$ is defined as

$$
C_{\operatorname{Imp}}=\left|\frac{\max \left(D_{1}\right)}{\mu\left(D_{1}\right)+1.5 \rho\left(D_{1}\right)}\right| \times 100 \%
$$

where $\max \left(D_{1}\right), \mu\left(D_{1}\right)$ and $\rho\left(D_{1}\right)$ are the maximum, mean and standard deviation of the wavelet decomposed signal $D_{1}$, respectively. The index of the critical level of harmonic

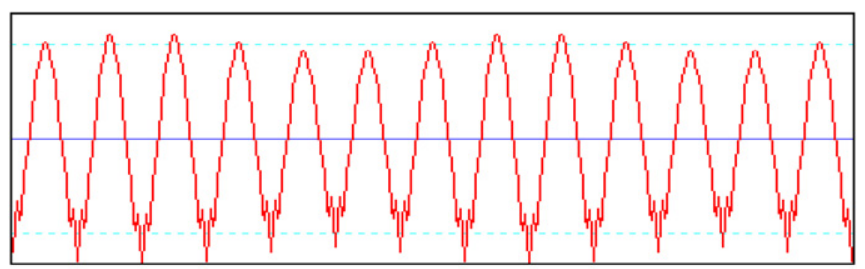

(a)

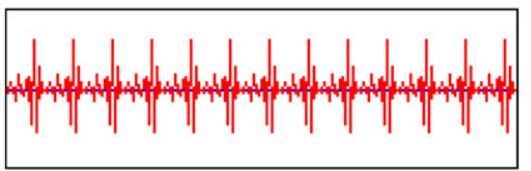

(b)

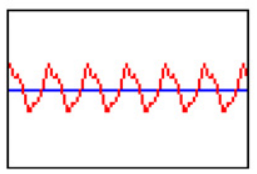

(c)

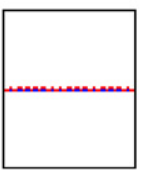

$(d)$

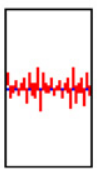

(e)

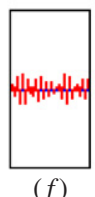

$(f)$
Figure 4. (a) An example of the waveform obtained from combining several harmonic distortions and voltage flicker, and its wavelet coefficients: (b) $D_{1} ;(c) D_{2} ;(d) D_{3} ;(e) D_{4} ;(f) S_{4}$.

distortions is the total harmonic distortion of the voltage $\left(V_{\text {THD }}\right)$, which can be formulated as follows [30]:

$$
V_{\mathrm{THD}}=\frac{\sqrt{\left|V_{2}\right|^{2}+\left|V_{3}\right|^{2}+\left|V_{4}\right|^{2}+\cdots}}{\left|V_{1}\right|} \times 100 \%,
$$

where $V_{1}$ is the amplitude of the fundamental voltage, and $V_{2}$, $V_{3}, V_{4}, \ldots, V_{n}$ are the amplitudes of the $2 \mathrm{nd}, 3 \mathrm{rd}, 4 \mathrm{th}, \ldots, n$th harmonic voltages, respectively.

$\Delta V_{10}$ is typically used to measure the voltage flicker, which is given by [19]

$$
\Delta V_{10}=\sqrt{\sum_{n}\left(a_{n} \cdot \Delta \overline{V_{n}}\right)^{2}},
$$

where $a_{n}$ is the visually sensible level of the $n$th harmonic frequency of the flicker and $\Delta \overline{V_{n}}$ is the peak-to-peak amplitude variance of the corresponding frequency of the flicker.

\section{Simulation tool and testing materials}

\subsection{Development of the GUI simulation tool}

The proposed classifier is implemented in an x86-based PC for demonstration. Figure 5 illustrates the layout of the graphic user interface (GUI) developed for the proposed classifier. The operating procedures are as follows:

(1) Select 'waveform generator' to generate the six types of commonly encountered PQ disturbance waveforms.

(2) Select 'rms estimator' to estimate the amplitude of the fundamental voltage and perform a four-scale hierarchical wavelet transform.

(3) Select 'recognition' to evaluate the wavelet coefficients and perform the power quality event classification.

The measured waveform is displayed in the middle of the GUI window. The dotted line and the dashed line of the waveform are the reference amplitude with 1 p.u. and the 


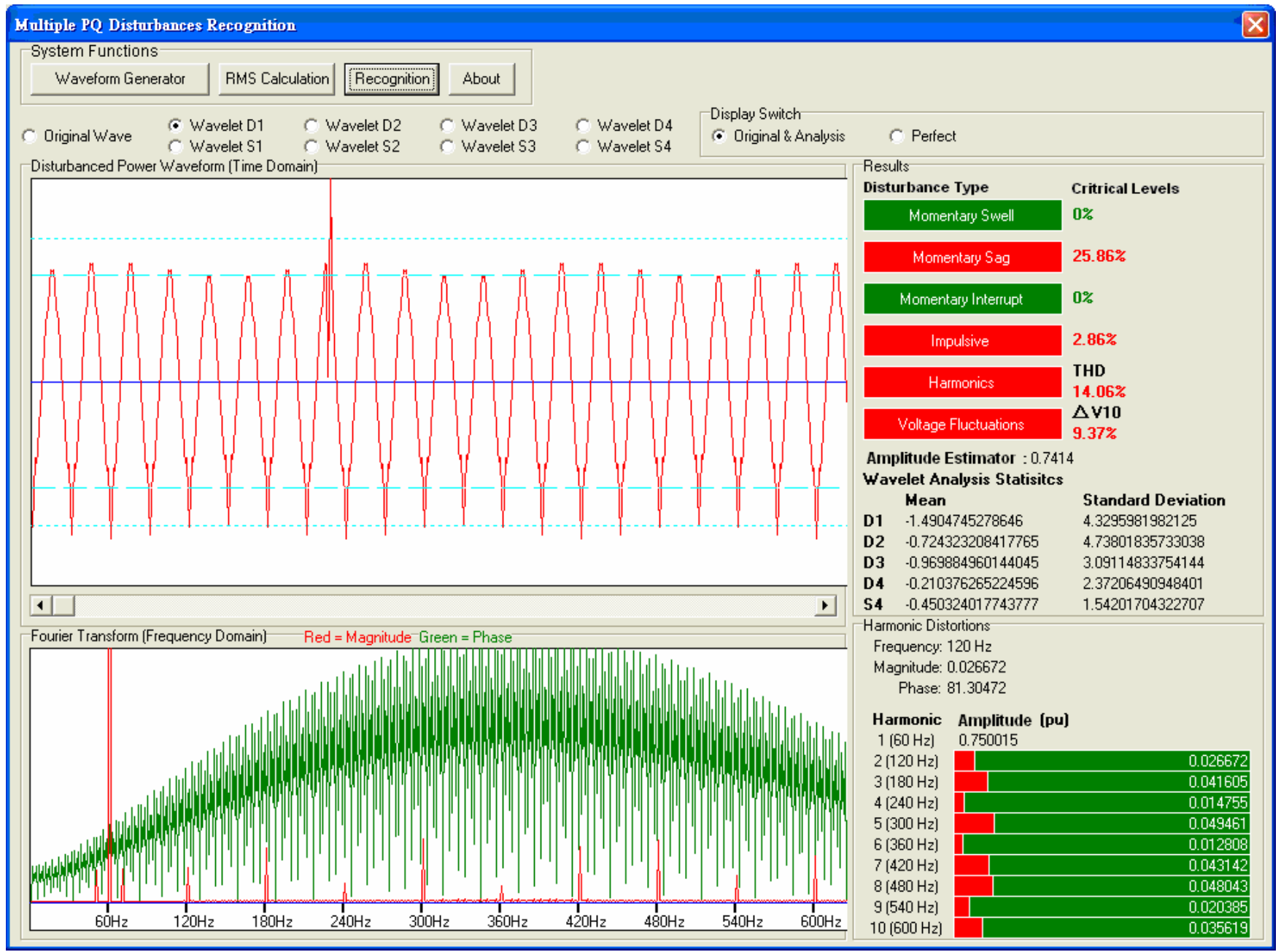

Figure 5. Layout of the demonstration program that comprises the proposed multiple power quality disturbance classifier and the harmonic analyzer.

estimated amplitude of the fundamental voltage, respectively. The recognition results of the proposed classifier are shown on the right side of the window. The types of detected disturbance are displayed with red color along with their corresponding critical level. A harmonic analyzer is also adopted, and displayed in the bottom of the window to provide more detailed information on the orders and amplitudes of the harmonics. The ideal sinusoidal wave with estimated amplitude and wavelet coefficients on each scale of the wavelet transform are displayed by selecting the radio buttons in the 'Display Switch' panel above the waveform displayer. Double clicking the waveform displayer block redraws the original disturbance waveform.

\subsection{Generation and collection of testing waveforms for performance evaluation}

The training data used in the training procedure are collected from field measurements and generated from the waveform generator implemented in the proposed algorithm. We used an oscilloscope to measure and record the electric signals (with fundamental frequency of $60 \mathrm{~Hz}$ ) at different locations in main engineering buildings at the campus area of Tamkang
University, Taipei, Taiwan around 01/2004 to 03/2005 to form the database of field measurements. The power waveform measurements were conducted directly from the power plugs of these main buildings. Power plugs may be located at several locations for each main building, e.g., near the elevator control room, distribution room, etc. The adopted oscilloscope was capable of measuring waveforms containing electric signals with frequency band up to hundreds of $\mathrm{MHz}$, so the mentioned various PQ disturbances can be accurately recorded. During field measurement for electric signals, the oscilloscope can save the waveforms on a floppy disc. Then, we put the floppy disc in computers to read the real power waveforms, and reduce the sampling rate of the measured data fed into the database.

However, measuring the waveforms of an actual PQ event is not only difficult but also requires advanced instruments. It is difficult to obtain a complete set of measurement data which cover all kinds of PQ disturbances to train and test the proposed classifier. Accordingly, this work develops a waveform generator that is embedded into the proposed algorithm and can generate commonly encountered PQ disturbances on a personal computer easily. 


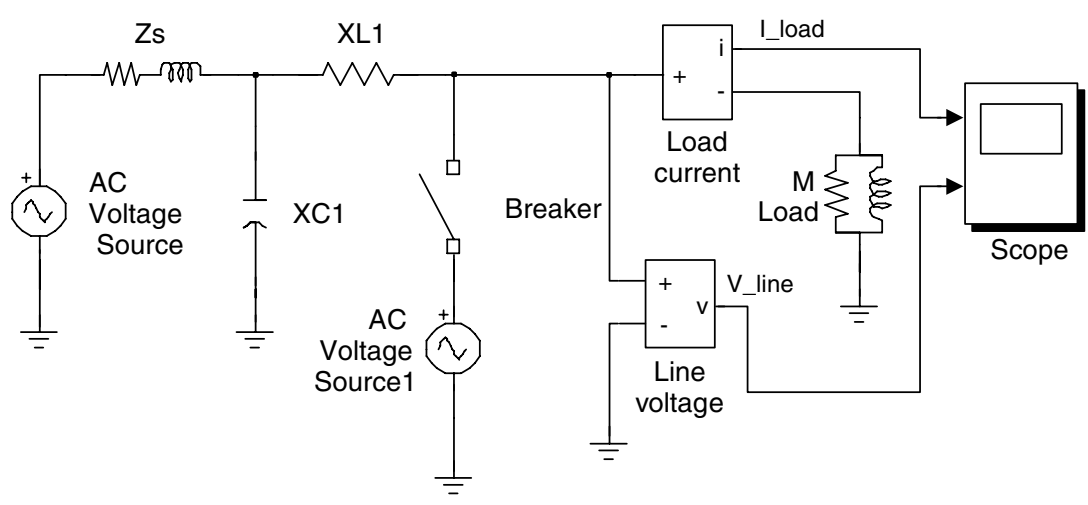

Figure 6. The Simulink circuit for simulating voltage swell.

This work utilizes two techniques to implement the waveform generator. One of them is a formula-based technique regardless of loadings in the electrical circuit. For example, waveforms of the voltage swell and sag can be generated by the equation

$$
V[n]=V_{s} \sin (S \omega / R) \pi,
$$

where $n$ denotes the number of the size of the generated waveform, $V_{s}$ represents the magnitude of the generated waveform, $S$ is the current sequence number of the sampling point, $\omega$ represents the fundamental frequency, $R$ symbolizes the sampling rate and $\pi$ represents the ratio of the circumference. $V_{s}$ is set as above $105 \%$ and below $90 \%$ of the rated value to generate the surge and sag [19], respectively.

The following equation illustrates how to build the waveforms regarding the voltage flicker, harmonic distortion and impulsive transient disturbance in the time domain:

$$
\begin{aligned}
& V(t)=V_{f} \sin \left(2 \pi \omega_{f} t\right) \cdot\left[V_{m} \sin (2 \pi \omega t)\right] \\
& \quad+\sum_{h} V_{h} \sin \left(2 \pi \omega_{h} t\right)+\Pi(x) \cdot V_{i} \sin \left(2 \pi \omega_{c} t\right) \cdot \operatorname{rand}(t),
\end{aligned}
$$

where $V_{f}, V_{m}, V_{h}$ and $V_{i}$ denote the amplitudes of the voltage flicker, fundamental voltage, harmonic distortion and impulsive transient disturbance, respectively. $\omega_{f}, \omega$ and $\omega_{h}$ are the frequencies of the voltage flicker, fundamental voltage and harmonics, respectively. $\Pi(x)$ is a boxcar function, which allows a periodical sinusoidal wave or random signals $\operatorname{rand}(t)$ to be added to the waveform for a small period at specific time $x$. By means of the boxcar function, we can issue an impulsive transient disturbance at a specific point of time by setting $\omega_{c}$ to $1 / 4$. On the other hand, we can issue a transient disturbance caused by capacitive switching via setting $\omega_{c}$ to a large value. In most cases, capacitive switching generates a high voltage impulse to power systems due to capacitor discharge. Therefore, in this study the transient disturbance caused by capacitive switching is defined in the category of impulsive transient disturbance.

The second technique for generating waveforms is based on an electrical circuit model. Two circuits programmed by MATLAB/Simulink can be used to illustrate this matter. Figure 6 is for simulating voltage swell disturbance where Zs denotes the system impedance, XL1 and XC1 represent the line impedance, $\mathrm{M}$ load symbolizes the loading and a branch consisted of the ac voltage source 1 and a breaker is utilized to simulate voltage swell disturbances in the power system. In that, the ac voltage source 1 and breaker are controlled for setting the magnitude and fault time of surge disturbances, respectively.

Figure 7 is designed for generating flicker in a power system. In figure 7 , ac voltage source represents the system voltage with rated amplitude and fundamental frequency (i.e., $60 \mathrm{~Hz}$ ) and $R$ represents the load impedance. The other two voltage sources, ac voltage sources 1 and 2 , are utilized to represent the flicker sources, which are with $20 \mathrm{~Hz}$ and $100 \mathrm{~Hz}$. These voltages can be expanded to $n$ sets for forming $n$ other flickers associated with the corresponding magnitude and frequency. In this study, we focus on power disturbances caused by flicker and/or harmonics, and the frequencies of harmonics are with multiples of fundamental frequency. As we know, an inter-harmonic is also a type of harmonic, and its frequency is a ratio of one harmonic and another. If an inter-harmonic exists in the power system, the amplitude of the fundamental frequency will fluctuate, and this will cause flicker [31, 32]. Therefore, in this study we treat interharmonic disturbance as flicker.

We collect training and test data sets using both types of waveform generators to comprehensively examine the performance of the proposed classifier. Six frequently encountered types of power quality disturbances are employed herein to train and test the proposed classifier. The six types of PQ disturbances are the voltage swell (type A), voltage sag (type B), voltage interrupt (type C), impulsive transient (type D), harmonics (type E) and voltage flicker (type F). Refer to IEEE Std 1159 [19] for the detailed definitions of these disturbances. The above proposed waveform generator can simulate all six types of PQ disturbance waveforms. In this work, three cases (groups) of composite PQ disturbances are utilized to train and test the developed classification algorithm. They are a single disturbance, dual disturbances and multiple disturbances.

- Single-disturbance case: a single type of PQ disturbance.

- Dual-disturbance case: two types of PQ disturbances mixed, of which one is randomly chosen from type A, type $\mathrm{B}$ or type $\mathrm{C}$, and the other is randomly chosen from type $\mathrm{D}$, type $\mathrm{E}$ or type $\mathrm{F}$. 


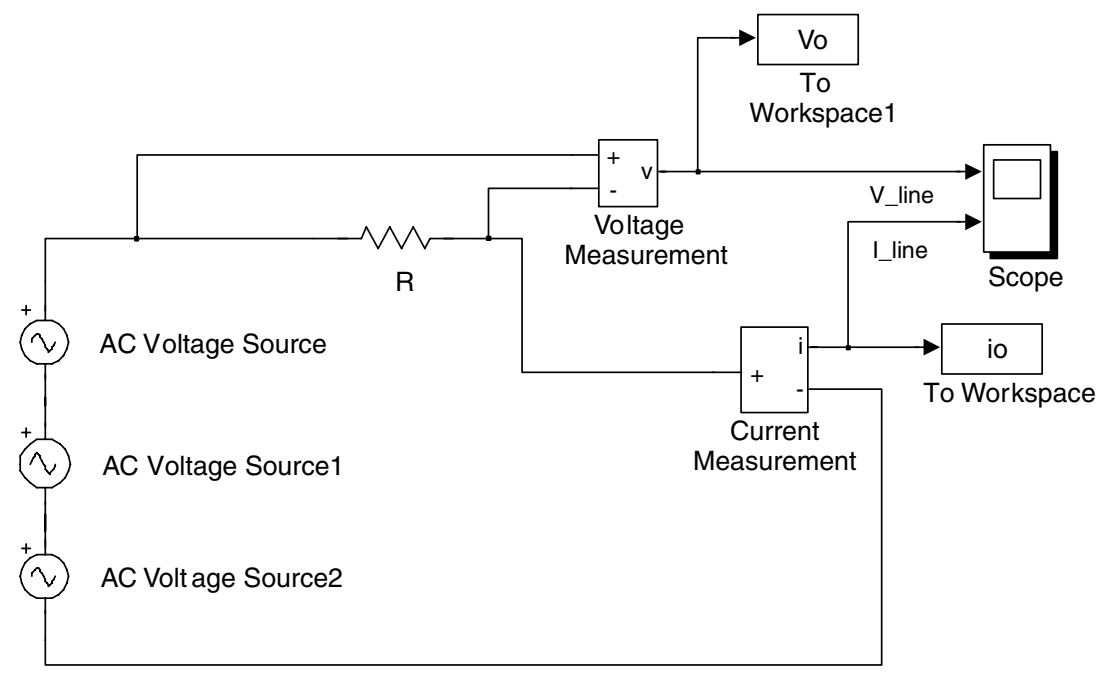

Figure 7. The Simulink circuit for simulating interrupts.

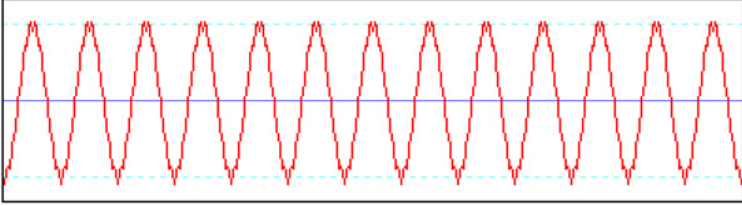

(a)

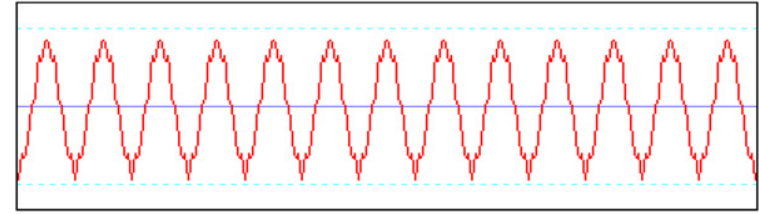

(b)

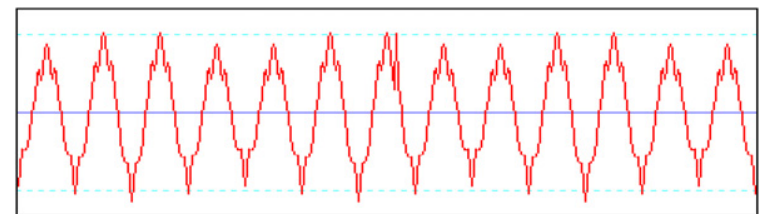

(c)

Figure 8. PQ disturbance cases: $(a)$ single disturbance; $(b)$ dual disturbances; $(c)$ multiple disturbances.

- Multiple-disturbance case: up to four types of PQ disturbances mixed, of which one is randomly chosen from type A, type B or type $\mathrm{C}$, and the others are randomly chosen from type $\mathrm{D}$, type $\mathrm{E}$ or type $\mathrm{F}$.

Figure 8 illustrates three examples of the waveforms for each group of disturbances: figure $8(a)$ contains the harmonic distortion; figure $8(b)$ is a combination of the voltage sag and harmonic distortion and figure $8(c)$ is a combination of the voltage sag, multiple harmonic distortions and voltage flicker. Critical levels of each type of disturbance in a waveform are calculated and then stored in the waveform database accompanying with the corresponding waveform according to equations (5)-(8).

The friendly GUI and waveform generator are embedded into the proposed multiple disturbances classifier to implement and integrated as a platform for various simulation experiments and educational demonstration.

\section{Experimental results}

This section presents the simulation results for the proposed classifier using electric signals that contain six typical types of disturbances. The disturbance waveforms are randomly generated and divided into training and testing sets. A big pool of samples is generated, including 1000 undistorted waveforms and 2000 distorted ones as the sample database. The sampling rate of the waveform is 30 points per cycle. The fundamental waveform has a frequency of $60 \mathrm{~Hz}$ with an amplitude of 1 p.u. (per unit).

The parameters of the ASNN are that the space preservation factor $\rho$ is set as 1.5 , the number of initially generating neurons is 50 and the activation function $\varphi_{o}$ is a hyperbolic tangent function. It is a nonlinear function widely utilized in neural network models because it provides neural classifiers with the ability to solve both linear and nonlinear problems, including the classification of PQ disturbances in the measured electric signals.

The accuracy used for the performance evaluation of the proposed multiple disturbances classifier is defined as

$$
\text { Accuracy }(\%)=\frac{\#\{\text { matched detection }\}}{\#\{\text { total disturbance types }\}} \times 100 \% \text {. }
$$

The proposed wavelet-based neural classifier was trained by these 3000 samples by 10 -fold cross validation. In other words, $90 \%$ of the sample database is the training set, and the rest $(10 \%)$ is the testing set to validate the training accuracy of the proposed classifier. During the training process, the number of neurons is rapidly increased when the training accuracy is low or bottlenecks are encountered. As depicted in figure 9, the amount of neurons in the utilized ASNN is successfully promoted. After six cycles of the reconfiguration process, the proposed classification classifier achieved $98.9 \%$ training accuracy in 120 iterations. 


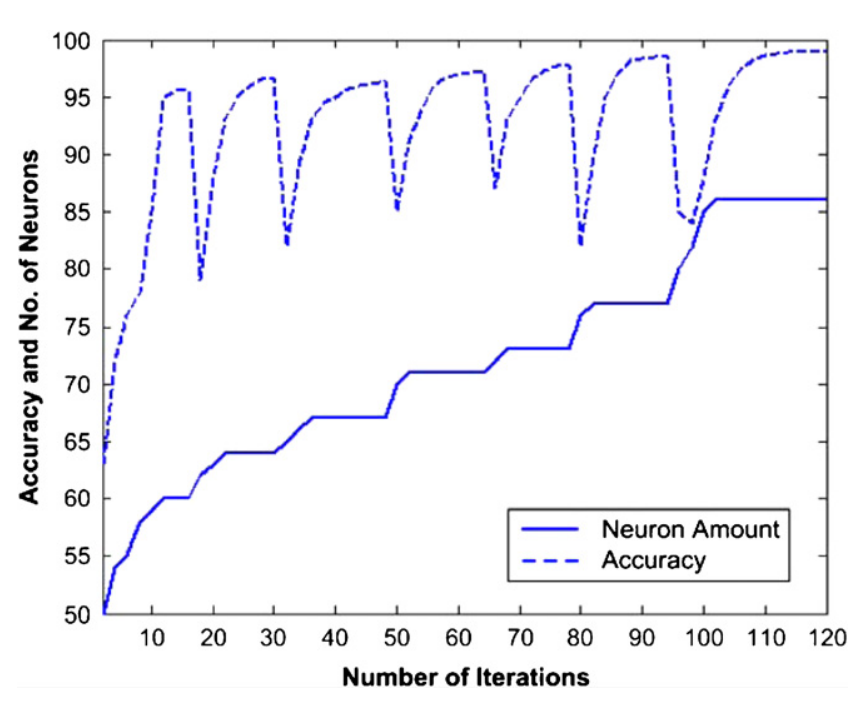

Figure 9. Evolutionary process of the training of the proposed classifier.

Table 1. Summarization of evaluation results (mean-square error).

\begin{tabular}{lllll}
\hline \multirow{2}{*}{ Simulation phases } & \multicolumn{4}{c}{ Critical level } \\
\cline { 2 - 5 } & $C_{\text {Amp }}$ & $C_{\text {Imp }}$ & $V_{\text {THD }}$ & $\Delta V_{10}$ \\
\hline Training & 0.0005 & 0.0035 & 0.0014 & 0.0008 \\
Testing & 0.0062 & 0.0097 & 0.0042 & 0.0023 \\
\hline
\end{tabular}

We set the initial number of hidden neurons to 50 in order to prevent the ASNN model from causing overfitting. The mean-square errors (MSE) of the predictions of each critical level yielded by the ASNN model on training and testing sets are summarized in table 1. The results show that the ASNN model can learn from testing waveforms, and predict the critical levels of disturbances of the newly measured waveforms. The evaluation results for the three test cases obtained from the proposed algorithm are described below.

\subsection{Single-disturbance case}

PQ disturbances can be easily recognized in the single disturbance case. Many available approaches at present can yield promising classification results in this kind of case as well. This study employs 750 disturbance waveforms to test the proposed classifier. The test results are summarized in table 2, which indicates that the proposed classifier can $100 \%$ discriminate all types of disturbances in the single disturbance case.

\subsection{Dual-disturbance case}

A total of 750 waveforms, consisting of two types of disturbances in a PQ event, are employed to test the performance of the proposed classifier. From table 2, the evaluation results show that the proposed classifier achieves an accuracy of $100 \%$ for the PQ disturbances of type C, type $\mathrm{E}$ and type $\mathrm{F}$. The accuracy rates for type A, type B and type $\mathrm{D}$ are $99.985 \%, 99.986 \%$ and $99.94 \%$, respectively.

An inspection of table 2 indicates that when the amplitude of the fundamental voltage is around 1.05 p.u. (near the threshold of the amplitude of the momentary swell disturbance) or 0.9 p.u. (near the threshold of the amplitude of the momentary sag disturbance), the impulsive transient disturbances might slightly influence the estimation precision of the amplitude estimator. The classifier may misclassify under such kinds of circumstances. Nevertheless, the classification accuracies of the proposed classifier with respect to the PQ disturbances of type A, type B and type D still reach pretty good results.

\subsection{Multiple-disturbance case}

This test case exploits the distinguishing characteristic of the proposed classifier. Most of the earlier methods are not capable of classifying a PQ event when it comprises up to four types of disturbances at the same time. An example case, as illustrated in figure $8(c)$, is one which contains numerous orders of harmonics and other disturbances.

From table 2, the simulation results reveal that the classification accuracy of the proposed classifier is still competent, especially for the PQ disturbances of types E and F. The proposed algorithm can maintain high accuracy for classifying harmonic distortions and the voltage flicker since the disturbance factors associated with voltage swell, sag, interrupt and transient impulsive disturbances are removed before the wavelet coefficients are fed into the ASNN classifier.

An inspection of the evaluation results shows that the classification accuracy of transient impulsive disturbances in the multiple-disturbance case is lower than the other cases, since when the electric signal contains higher orders of harmonic distortions, it would make the impulse detector in equation (4) slightly insensitive to the impulsive transient disturbances. However, the achieved classification accuracy for transient impulsive disturbances is still as high as $96.8 \%$ in this case.

Table 2. Summarization of evaluation results (accuracy) of the proposed PQ classifier.

\begin{tabular}{lllllll}
\hline & \multicolumn{6}{c}{ Accuracy $^{\mathrm{a}}$} \\
\cline { 2 - 7 } Simulation cases & Type A & Type B & Type C & Type D & Type E & Type F \\
\hline Single disturbance & $100 \%$ & $100 \%$ & $100 \%$ & $100 \%$ & $100 \%$ & $100 \%$ \\
Dual disturbances & $99.99 \%$ & $99.99 \%$ & $100 \%$ & $99.94 \%$ & $100 \%$ & $100 \%$ \\
Multiple disturbances & $98.6 \%$ & $98.7 \%$ & $99.2 \%$ & $96.8 \%$ & $99.8 \%$ & $99.5 \%$ \\
\hline
\end{tabular}

${ }^{a}$ Accuracy of classification is defined in equation (9). 


\section{Conclusions}

This work proposes a pipeline-based classifier integrating the rms voltage amplitude estimator, wavelet transform, and ASNN to recognize multiple types of disturbances in a PQ event of power systems. The theoretical foundation and implementation of the PQ events classifier are described in this paper. Six types of PQ disturbances are considered in this work. They are momentary swell disturbances, momentary sag disturbances, momentary interrupt disturbances, impulsive transient disturbances, harmonic distortions and voltage flicker. The first three types are identified in the time domain and the rest are identified in the wavelet domain.

A wave generator for producing simulation samples is also developed. Three thousand testing waveforms are used to evaluate the performance of the developed classifier. The testing results manifest that the proposed classifier can recognize multiple types of $\mathrm{PQ}$ disturbances in a PQ event, and achieve classification accuracy higher than $99 \%$ both in the single-disturbance and dual-disturbance cases. In the multiple-disturbance case, the classification accuracy of more than $96.8 \%$ is achieved for the impulsive transient disturbance, around $98.7 \%$ for momentary swell and sag disturbances and $99 \%$ for harmonic distortions and voltage flicker.

\section{Acknowledgments}

The authors would like to thank the National Science Council of the Republic of China for financially supporting this research under Contracts: NSC 94-2213-E-002-120 and NSC 94-2213-E-032-029.

\section{References}

[1] Santoso S, Powers E J, Grady W M and Parsons A C 2000 Power quality disturbance waveform recognition using wavelet-based neural classifier: part 1. Theoretical foundation IEEE Trans. Power Deliv. 15 222-8

[2] Santoso S, Powers E J, Grady W M and Parsons A C 2000 Power quality disturbance waveform recognition using wavelet-based neural classifier: part 2. Application IEEE Trans. Power Deliv. 15 229-35

[3] Kaewarsa S and Attakitomongcol K 2006 Wavelet-based neural network for power quality disturbance recognition and classification Aust. J. Electr. Electron. Eng. 3 67-76

[4] Kaewarsa S, Attakitomongcol K and Kukworawanichpong $\mathrm{T}$ 2007 Recognition of power quality events by using multiwavelet-based neural network Proc. 6th IEEE/ACIS Int. Conf. on Computer and Information Science pp 993-8

[5] Attakitomongcol K, Hardin D P and Wilkes D M 2001 Multiwavelet prefilters-parts II: optimal orthogonal prefilters IEEE Trans. Image Process. 10 1476-87

[6] Chung J, Powers E J, Grady W M and Bhatt S C 2002 Power distribution classifier using a rule based method and wavelet package-based hidden Markov model IEEE Trans. Power Deliv. 17 233-41

[7] Santoso S, Grady W M, Powers E J, Lamoree J and Bhatt S C 2000 Characterization of distribution power quality events with Fourier and wavelet transforms IEEE Trans. Power Deliv. 15 247-54

[8] Zhu T X, Tso S K and Lo K L 2004 Wavelet-based fuzzy reasoning approach to power-quality disturbance recognition IEEE Trans. Power Deliv. 19 1928-35
[9] Dash P K and Chilukuri M V 2004 Hybrid S-transform and Kalman filtering approach for detection and measurement of short duration disturbances in power networks IEEE Trans. Instrum. Meas. 53 588-96

[10] He H and Starzyk J A 2006 A self-organizing learning array system for power quality classification based on wavelet transform IEEE Trans. Power Deliv. 21 286-95

[11] Shin Y J, Powers E J, Grady M and Arapostathis A 2006 Power quality indices for transient disturbances IEEE Trans. Power Deliv. 21 253-61

[12] Gerek O N and Ece D G 2006 Power-quality event analysis using higher order cumulants and quadratic classifiers IEEE Trans. Power Deliv. 21 883-9

[13] Gerek O N and Ece D G 2005 An adaptive statistical method for power quality analysis IEEE Trans. Instrum. Meas. 54 184-91

[14] Eldery M A, El-Saadany E F, Salama M M A and Vannelli A 2006 A novel power quality monitoring allocation algorithm IEEE Trans. Power Deliv. 21 768-77

[15] Tey L H, So P L and Chu Y C 2005 Improvement of power quality using adaptive shunt active filter IEEE Trans. Power Deliv. 20 1558-68

[16] Won D J, Ahn S J and Moon S I 2005 A modified sag characterization using voltage tolerance curve for power quality diagnosis IEEE Trans. Power Deliv. 20 2638-43

[17] Kezunovic M and Liao Y 2002 A novel software implementation concept for power quality study IEEE Trans. Power Deliv. 17 544-9

[18] Zhou X, Zhou C and Kemp I J 2005 An improved methodology for application of wavelet transform to partial discharge measurement denoising IEEE Trans. Dielectr. Electr. Insul. 12 586-94

[19] IEEE Std 1159-1995 1993 IEEE Recommended Practice for Monitoring Electric Power Quality (New York: IEEE Press)

[20] Chen M T and Sakis Meliopoulos A P 2002 A hybrid digital algorithm for harmonic and flicker measurements Proc. 2002 IEEE Power Engineering Society Winter Meeting pp 1488-93

[21] Antonia P S 2003 Applications in Time-Frequency Signal Processing (Boca Raton, FL: CRC Press)

[22] Burrus C S, Gopinath R A and Guo H 1997 Introduction to Wavelets and Wavelet Transforms: A Primer (Englewood Cliffs, NJ: Prentice Hall)

[23] Chui C K 1992 An Introduction to Wavelets (San Diego, CA: Academic)

[24] Ganis M D, Wilson C L and Blue J L 1998 Neural network-based systems for handprint OCR applications IEEE Trans. Image Process. 7 1097-112

[25] Osowski S, Linh T H and Brudzewski K 2004 Neuro-fuzzy network for flavor recognition and classification IEEE Trans. Instrum. Meas. 53 638-44

[26] Antognetti P and Milutinovic V 1991 Neural Networks Concepts, Applications, and Implementation vol I (New Jersey: Prentice Hall)

[27] Antognetti P and Milutinovic V 1991 Neural Networks Concepts, Applications, and Implementation vol II (New Jersey: Prentice Hall)

[28] URL: http://bem.bime.ntu.edu.tw/clchuang/ASNN.pdf

[29] Sankaran C 2001 Power Quality (Boca Raton, FL: CRC Press)

[30] IEEE Std 519-1992 1992 IEEE Recommended Practice for Monitoring Electric Power Quality (New York: IEEE Press)

[31] Zhang D, Xu W and Nassif A 2005 Flicker source identification by inter-harmonic power direction Proc. Canadian Conf. on Electrical and Computer Engineering pp 549-52

[32] Keppler T, Watson N R, Arrillaga J and Chen S 2003 Theoretical assessment of light flicker caused by sub- and inter-harmonic frequencies IEEE Trans. Power Deliv. 18 329-33 